\title{
Intralesional Measles Mumps Rubella vaccine versus BCG in the treatment of multiple cutaneous warts: a comparative study
}

\section{Gopal Nayana, Nirvanappa Vinay, Rangaswamy Umadevi, Rangegowda Suresh, Nagesha Parvathi}

Department of Dermatology Venereology and Leprosy, Hassan Institute of Medical Sciences, Karnataka, India

Corresponding author: Nagesha Parvathi, MD, E-mail: drparvathicn@gmail.com

\begin{abstract}
:
Background: Verrucae or warts are benign epithelial proliferations that occur due to accumulation of keratinocytes induced by HPV. Immunotherapy is a new promising modality which uses the principle of activation of one's own immunity against the disease. Materials and methods: The study included 40 patients with multiple cutaneous warts in two groups of 20 each. Assigned treatment was injected into the single largest wart at an interval of 3 weeks until complete clearance or for a maximum of 3 times. Results: A statistically significant difference $(\mathrm{p}=0.018)$ was seen in the therapeutic response when compared to that of the BCG. All 20(100\%) patients in the MMR group showed $>50 \%$ clearance while in the BCG group 14(70\%) patients showed $>50 \%$ clearance according to Physician's Global Assessment scores. Conclusions: Intralesional immunotherapy with MMR and BCG vaccines are both safe and promising whereas the therapy with MMR stands out with statistically significant results.
\end{abstract}

Key words: Warts; Verruca; Immunotherapy; MMR; BCG; Intralesional

\section{INTRODUCTION}

Cutaneous warts or verrucae are benign epidermal proliferations caused by Human Papilloma virus [1]. Diverse HPV strains are responsible for specific types of warts. Prevalence in the general population is found to be $7-12 \%$ [2]. Different forms of cutaneous warts include common warts (verruca vulgaris), palmar and plantar warts (verrucae palmares et plantares), mosaic warts, flat warts (verrucae planae), butcher's warts, filliform warts, anogenital warts(condyloma accuminata) and Buschke - Lowenstein tumor. In general, classification of warts is based on morphology, histology, and anatomic location. Though most of them are known to be self-resolving, it takes around 1-2 years for the complete resolution. Along with the long term cosmetic embarrassment, patients also complain of a certain amount of pain and discomfort. Different treatment modalities most of which are ablative have been tried over time such as electrocautery, chemical cautery, cryotherapy, carbon dioxide laser ablation, surgical curettage and topical keratolytics. Treating these warts becomes challenging when they are multiple, recalcitrant or recurrent. So, to address this the immunogenic potential of certain vaccines and chemicals like Diphenylcyclopropenone, Squaric acid dibutyl ester, Tuberculin, Candida antigen, HPV vaccine, Purified Protein Derivative, BCG, Measles Mumps Rubella vaccine have all been explored. This study was undertaken with an intention to compare the safety and efficacy of the two immunotherapies Measles Mumps Rubella vaccine versus BCG in treating multiple verrucae vulgaris [3].

Intralesional immunotherapy employs the ability of the immune system to recognize viral antigens and induce a delayed-type hypersensitivity reaction(by recruiting CD4 $\mathrm{T}$ cells and macrophages), also recruits

\footnotetext{
How to cite this article: Nayana G, Vinay N, Umadevi HR, Suresh MR, Parvathi N. Intralesional Measles Mumps Rubella vaccine versus BCG in the treatment of multiple cutaneous warts: a comparative study. Our Dermatol Online. 2021;12(e):e82.

Submission: 02.02.2021; Acceptance: 08.07.2021

DOI: 10.7241 /ourd.2021e.82
} 
macrophages and NK cells, thereby increasing the ability of the immune system to recognize and clear HPV. Consequent to this, the stimulated immune response clears all lesions on other body sites along with locally treated lesions [3].

\section{MATERIALS AND METHODS}

The study was designed as a single centre, doubleblind, randomized, parallel group, comparative study. Clearance from the Institutional Ethics Committee was obtained before the start of the study and written informed consent was obtained from all study participants. All consecutive patients of either sex suffering from clinically diagnosed cutaneous warts and having more than one wart attending the dermatology outpatient department of Hassan Institute of Medical Sciences, Hassan were included. Pregnant or lactating women, children below 12 years, patients suffering from immunosuppression due to drug or disease, those with mucosal warts, non-consenting patients, patients on any other treatment for warts and those having fever or any signs of inflammation/infection were excluded.

A total of 40 patients were recruited after taking a written consent from them. They were divided in two groups by simple random sampling using a lottery method.

Group A: MMR group -20 patients to be given with $0.1 \mathrm{ml}$ or less of intralesional MMR (into the largest verruca)

Group B: BCG group - 20 patients to be given with o. $1 \mathrm{ml}$ or less of intralesional BCG (into the largest verruca)

A thorough clinical examination for identification of the characteristics of the warts including site, size, number and presence or absence of distant warts were done and documented. Photographs of the lesions at the first visit, and then at subsequent sessions were taken.

Only the single largest wart was injected using an insulin syringe, and the injection was given at 3 weekly interval, until clearance or for a maximum of three treatment sessions, whichever is earlier. Clinical (Table 1), and photographic assessment to look for the improvement or any side effects was done at every visit.

The clinical response using Physician Global Assessment(PGA)was evaluated as follows:
Table 1: Patient self assessment score elaborated Patient satisfaction level Score on PSA

Remarkable improvement

Marked improvement

Moderate improvement

Minimal improvement

None/ No improvement

1) Grade 0: No improvement

2) Grade 1: Mild improvement ( $\leq 25 \%$ reduction in size)

3) Grade 2: Moderate improvement (26-50\% reduction in size)

4) Grade 3: Marked improvement (51 -75\% reduction in size)

5) Grade 4: Near total or total improvement $(\geq 76 \%$ reduction in size).

Patient satisfaction level was assessed using Patients' Self Assessment (PSA) scale as (Table 1).

\section{Ethics Statement}

A clearance certificate was obtained from the Instituitional Ethical Committee before start of the study.

\section{RESULTS}

A total of 40 patients were recruited in the study. 20 each were assigned to two treatment groups. Out of 40 patients, all of them completed the study.

Patients in the MMR group were in the age group ranging from $18-72$ years with a mean of 28.25 years; while the patients in BCG group belonged to the age group of $18-49$ years with a mean of 25.25 years; In the MMR group there were $16(80 \%)$ males and $4(20 \%)$ females, whereas in the BCG group, there were $13(65 \%)$ males and 7 (35\%) females(Table 2). With respect to occupation, MMR group had 14 (70\%) students, and $2(10 \%)$ each of farmers, businessmen and homemakers, while the BCG group had 13(65\%) students, $2(10 \%)$ homemakers and 1 each of auto driver, electrician, farmer, nurse and teacher.

According to the Physician's Global Assessment(PGA) scale which ranged from 0-4, at the end of the study, in the MMR group, 14 (70\%) patients showed 51-75\% reduction in size (Grade 3 ) and 6(30\%) patients showed 26-50\% reduction in size (Grade 2) (Table 3 ). Whereas in the BCG group, 9 (45\%) patients showed 
$51-75 \%$ reduction in size (Grade 3), 5(25\%) patients showed 26-50\% reduction in size (Grade 2) and $6(30 \%)$ patients showed $1-25 \%$ reduction in the size (Grade 1$)$; (Table 4); (Figure 1).

Similarly according to the Patients' Self Assessment (PSA) score, in the MMR group, 15 (75\%) patients showed remarkable (Grade 4) improvement, 4 (20\%) patients showed marked (Grade 3) improvement and $1(5 \%)$ patient showed moderate (Grade 2) improvement (Table 3). Whereas in the BCG group, 9(45\%) patients showed remarkable (Grade 4) improvement, 1 (5\%) patient showed marked (Grade 3) improvement, $7(35 \%)$ patients showed moderate (Grade 2) improvement and 3 (15\%) patients showed minimal (Grade 1) improvement (Table 4); (Figure 2).

Table 2: Baseline characteristics

\begin{tabular}{lcc}
\hline CHARACTERISTICS & GROUP A & GROUP B \\
\hline Age (in years) & $18-72$ & \\
Range & 28.25 & $18-49$ \\
Mean & & 25.25 \\
Sex & 4 & 7 \\
Female & 16 & 13 \\
Male & $1: 4$ & $1: 0.54$ \\
F:M & $3-19$ & $3-48$ \\
Number of lesions(range) & $3-24$ months & $3-18$ months \\
Duration(range) & & \\
Distribution & 2 & 0 \\
A & 12 & 12 \\
B & 1 & 5 \\
C & 2 & 8 \\
D & 3 & 1 \\
E & 1 & 3 \\
F & 4 & 2 \\
G & 1 & 0 \\
H & 0 & 1 \\
I & & \\
\hline
\end{tabular}

A - Periungual area, B - Dorsum of hands, C - Forearm, D - Dorsum of feet, E - Legs, F - Face, G - Palms, H - Axilla, I - Inguinal crease

Table 3: Group 1(MMR group)- Assessment at 9 weeks

\begin{tabular}{lccccc}
\hline \multicolumn{5}{c}{ Physician's Global Assessment(PGA) } \\
\hline $\begin{array}{l}\text { Grades } \\
\text { putients of }\end{array}$ & Grade 4 & Grade 3 & Grade 2 & Grade 1 & Grade 0 \\
\hline \multicolumn{5}{c}{ Patients' Self Assessment(PSA) } \\
\hline $\begin{array}{l}\text { Grades } \\
\text { pumber of } \\
\text { patients }\end{array}$ & Grade 4 & Grade 3 & Grade 2 & Grade 1 & Grade 0 \\
\hline
\end{tabular}

Table 4: Group 2(BCG group) - Assessment at 9 weeks

\begin{tabular}{lccccc}
\hline \multicolumn{5}{c}{ Physician's Global Assessment(PGA) } \\
\hline Grades & Grade 4 & Grade 3 & Grade 2 & Grade 1 & Grade 0 \\
\hline $\begin{array}{l}\text { Number of } \\
\text { patients }\end{array}$ & 0 & 9 & 5 & 6 & 0 \\
\hline \multicolumn{5}{c}{ Patients' Self Assessment(PSA) } \\
\hline $\begin{array}{l}\text { Grades } \\
\text { patients }\end{array}$ & Grade 4 & Grade 3 & Grade 2 & Grade 1 & Grade 0 \\
\hline
\end{tabular}

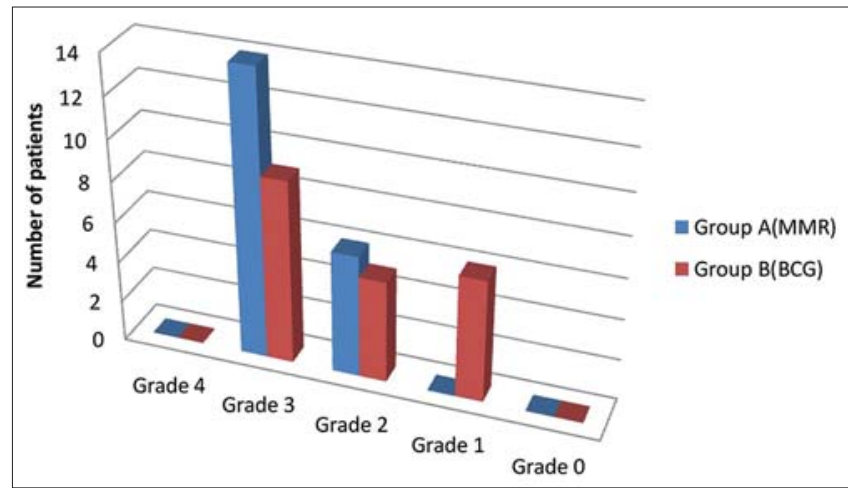

Figure 1: Graphical representation of Physician's Global Assessment at week 9 .

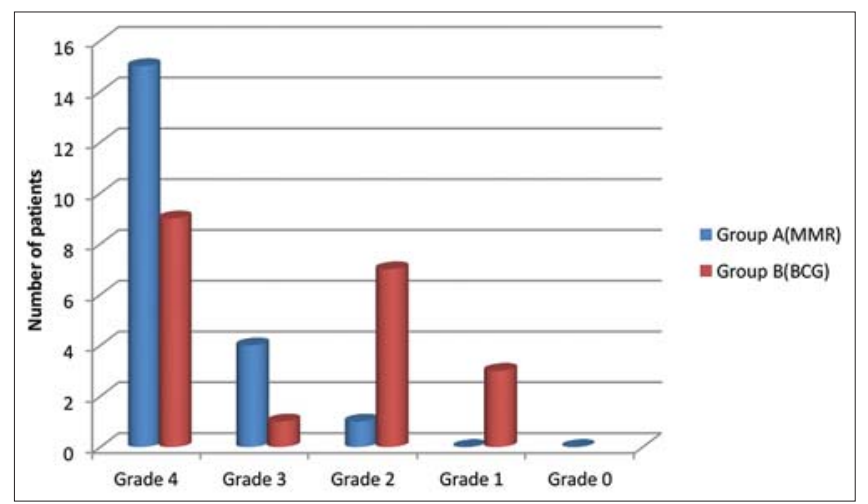

Figure 2: Graphical representation of Patient's Self Assessment score at week 9 .

\section{Side Effect Profile}

Local side effects like pain and erythema were noted in 8 of the patients in the BCG group which subsided on the third day of injection. Flu like symptoms were noted in two patients which on treatment with Paracetamol, subsided on second day of injection. There were no adverse effects in the MMR group.

\section{DISCUSSION}

Treatment of multiple cutaneous warts has been a therapeutic challenge for every physician. Immunotherapy has emerged as a promising modality for treating them in the recent years. Although several modalities have been tried, a universally acceptable, most efficacious and safer agent is yet to be studied and certified.

In 2010, Nofal and Nofal [4] conducted a case control study taking 135 patients with single and multiple common warts giving intralesional MMR vaccine to the first group and intralesional normal saline to the second group. They found that a 
highly significant difference was found between the therapeutic response of warts to MMR vaccine and saline control group. In the MMR group, complete response was achieved in $80 \%$ and $84.6 \%$ of patients presenting with recalcitrant and multiple warts respectively. Comparing the above mentioned study with the results of MMR group in our study, $70 \%$ of the patients achieved complete remission according to Physicians Global Assessment whereas 90\% of the patients showed remarkable satisfaction at the end of the treatment. And there was a significantly higher grade of improvement in the MMR group when compared with the BCG treated group. This was inferior to the study by Nofal and Nofal probably due to the lower sample size.

In 2019, in a study by Jaisinghani et al [5] BCG immunotherapy was used for recurrent multiple warts. They found that complete clearance was seen in $70 \%$ of the patients. Whereas in the present study using BCG on one of the study groups, complete clearance was seen in none according to Physician,s Global Assessment and $45 \%$ of the patients showed remarkable satisfaction. The resuts obtained here were inferior to the above mentioned study.

In a study by Munnangi et al [6], they have compared the efficacy of MMR vs BCG given intralesionally in multiple warts, taking 15 patients each in two groups treated with either vaccines. They noted that there was statistically significant difference both at lesional site and distant site where higher efficacy was seen with MMR compared to BCG - complete clearance $11(73.3 \%)$ vs $5(33.3 \%)$ respectively, partial clearance $3(20 \%)$ vs $4(26.7 \%)$ respectively and no response $1(6.7 \%)$ vs $6(40 \%)$ respectively. The results of this study is similar to that obtained in our study where MMR showed significantly higher efficacy when compared to BCG - complete clearance was seen in $14(70 \%)$ vs $9(45 \%)$ respectively, partial clearance was seen in $6(30 \%)$ vs 11 (55\%) respectively and no response was seen in none. MMR has shown higher efficacy over BCG in both the studies, whereas the percentage of complete clearance is less in our study probably due to the shorter follow-up period. It is also worth noting that, none in our study is left with no response.

The side effects observed in this study were very minimal and subsided by third day of treatment, so both the modalities were found to be safe.

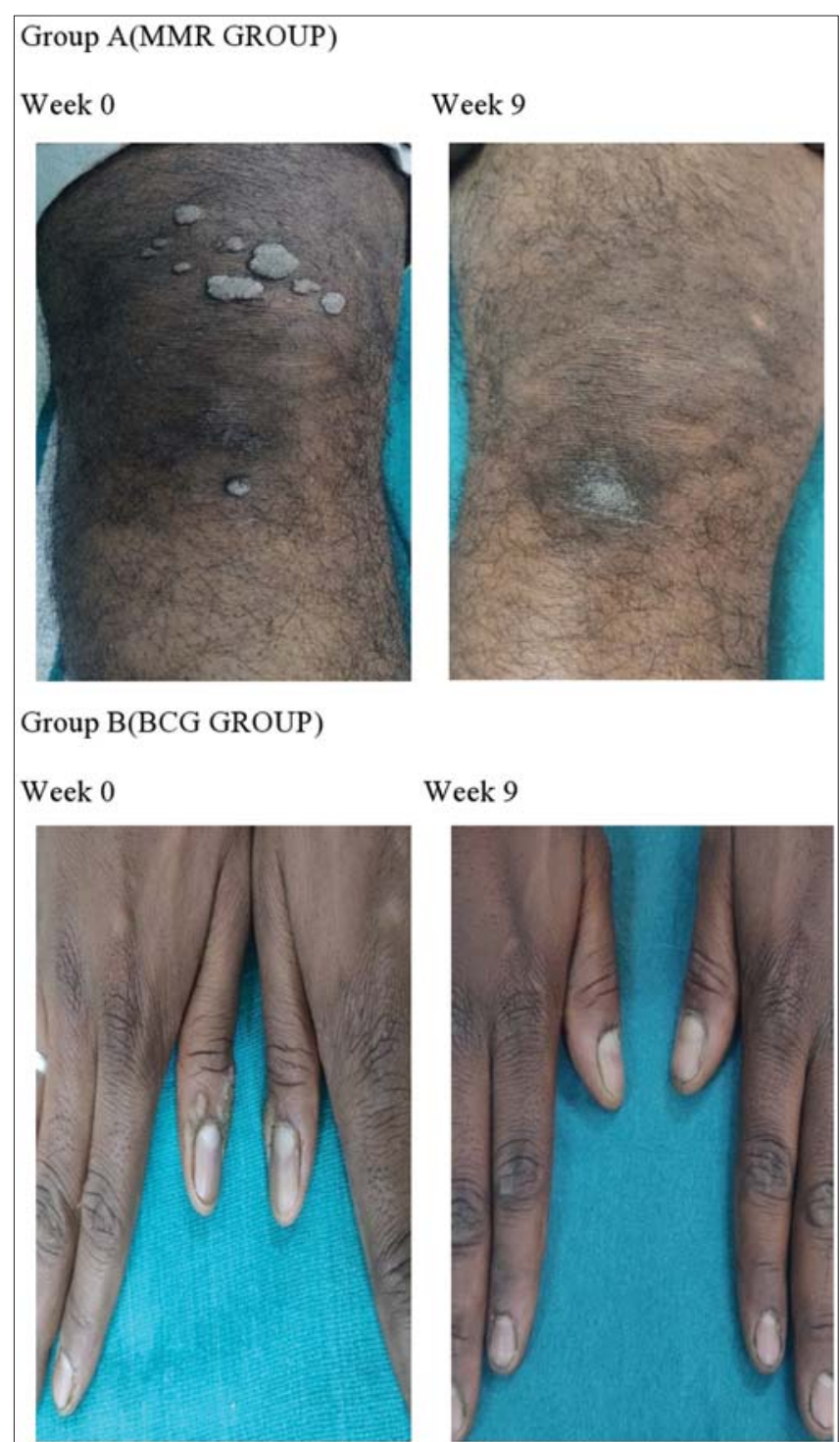

Figure 3: Treatment outcome at 9 weeks respectively in MMR and BCG groups.

\section{CONCLUSION}

Both intralesional MMR and BCG are safe and effective in treating multiple cutaneous warts (Figure 3). And among the two agents compared, MMR vaccine given intralesionally showed better efficacy than that of BCG. Hence it can be concluded that intralesional MMR vaccine is a safer, cost effective, efficacious and promising modality in treating multiple cutaneous warts. Also, future studies with higher sample size and case control type would be required to ascertain the same.

\section{Statement of Human and Animal Rights}

All the procedures followed were in accordance with the ethical standards of the responsible committee on human experimentation 
(institutional and national) and with the 2008 revision of the Declaration of Helsinki of 1975.

\section{Statement of Informed Consent}

Informed consent for participation in this study was obtained from all patients.

\section{REFERENCES}

1. Mulhem E, Pinelis S. Treatment of nongenital cutaneous warts. Am Fam Physician. 2011;84:288-93.

2. Lynch MD, Cliffe J, Morris-Jones R. Management of cutaneous viral warts. BMJ. 2014;348:1-6.

3. Podder I, Bhattacharya S, Mishra V, Sarkar TK, Chandra S, Sil A, et al. Immunotherapy in viral warts with intradermal Bacillus Calmette-Guerin vaccine versus intradermal tuberculin purified protein derivative: A double-blind, randomized controlled trial comparing effectiveness and safety in a tertiary care center in Eastern I. Indian J Dermatol Venereol Leprol. 2017;83:411.

4. Nofal A, Nofal E. Intralesional immunotherapy of common warts: successful treatment with mumps, measles and rubella vaccine. J Eur Acad Dermatol Venereol. 2010;24:1166-70.

5. Jaisinghani AK, Dey VK, Suresh MS, Saxena A. Bacillus Calmetteguerin immunotherapy for recurrent multiple warts: an open-label uncontrolled study. Indian J Dermatol. 2019;64:164.
6. Munnangi P, Kishore JCKLP, Devi VN. Comparative study between intralesional MMR and intralesional BCG in treatment of verruca vulgaris. 2018;17:44-50.

7. Shaker ESE, Doghim NN, Hassan AM, Musafa SS, Fawzy MM. Immunotherapy in cutaneous warts: comparative clinical Study between MMR vaccine, tuberculin, and BCG Vaccine. J Cosmet Dermatol. 2021 Jan 6. doi: 10.1111/jocd.13921.

8. Ebrahim HM, Asaad AM, El desoky F, Morsi HM. Bacillus Calmette-Guerin polysaccharide nucleic acid vs Bacillus CalmetteGuerin vaccine in the treatment of warts: A comparative, doubleblind, controlled study. Dermatol Ther. 2021;34:e14549.

9. Rao AG, Haqqani R. Study of BCG immunotherapy in the management of multiple, extensive non-genital cutaneous common warts. Indian Dermatol Online J. 2020;11:784-8.

10. Gogia P, Thami GP, Poonia K, Bhalla M, Garg G. Efficacy of tuberculin immunotherapy in verruca vulgaris: our experience from single center from North-West India and review of literature. Dermatol Ther. 2021;34:e14843

11. Nofal A, El-Arab RE, Nasr M, Alakad R. Intralesional measles, mumps, and rubella vaccine versus intralesional candida antigen in the treatment of common and plantar warts. J Cutan Med Surg. 2021 Feb 3;1203475421991130.

Copyright by Gopal Nayana, et al. This is an open access article distributed under the terms of the Creative Commons Attribution License, which permits unrestricted use, distribution, and reproduction in any medium, provided the original author and source are credited.

Source of Support: Nil, Conflict of Interest: None declared. 